\title{
Expiratory flow limitation in mechanical ventilation: a model study of lung non-homogeneities
}

\author{
C. Brighenti ${ }^{1}$, P. Barbini ${ }^{2}$, G. Cevenini ${ }^{2}$ \& G. Gnudi ${ }^{1}$ \\ ${ }^{I}$ Department of Electronics, Computer Science and Systems, \\ Bologna University, Cesena, Italy \\ ${ }^{2}$ Department of Surgery and Bioengineering, Siena University, Italy
}

\begin{abstract}
A non linear dynamic morphometric model of the human lung, based on Weibel's symmetrical model, was recently proposed for studying expiratory flow limitation (EFL) during mechanical ventilation in normal and obstructive conditions. The model is modified to study what happens when only a certain part of the lungs is affected by an obstructive pulmonary condition. In particular we consider the two main bronchi separately and, starting from each main bronchus, we divide each lung into two distinct regions with different geometrical and mechanical characteristics. In each region we assume that all branches of a certain generation are identical. The obstructive condition is characterized by increasing the respiratory zone resistance and by decreasing the airway section of deeper generations (from 8 to 16). We also changed the transmural pressure-diameter curves of these generations to account for easier collapsibility of these airways. The latter changes are applied to (a) the pathological region/s only or (b) all four regions. In case (a) we observed that EFL is not present until the pathology affects all the regions. In case (b) moderate EFL was evident even when only $25 \%$ of the lungs were involved and increased with increasing percentage of involvement. From this preliminary study, therefore, EFL seems to develop only if distinct parts of the conductive zone become significantly collapsible.
\end{abstract}

Keywords: breathing mechanics, expiratory flow limitation, mechanical ventilation, morphometric model, lung non-homogeneities. 


\section{Introduction}

A modelling approach can be useful to interpret and explain pathological modifications of the bronchial tree producing flow limitation in critical patients during mechanical ventilation. In particular, detailed morphometric models provide insights into this phenomenon, since they describe the nonlinear behaviour of different generations of the bronchial tree and quantify the geometrical modifications occurring inside the airways during a tidal breath.

Previous studies demonstrated that airway non-homogeneous constriction occurs in several respiratory diseases [1-3]. Moreover, a recent paper showed that airway non-homogeneity can help to understand flow limitation in a normal subject during maximum forced expiratory manoeuvres [4]. The model proposed by Polak and Lutchen [4], like the Lambert model [1], is quasi-static, even though it considers the main non linear mechanisms involved in expiratory flow limitation. The other proposed models are dynamic but linear [2-3].

We recently developed a homogeneous model of the human lung that is dynamic and, at the same time, implements various non linear properties of airways [5]. This model, based on the Weibel symmetrical morphometric description of the tracheobronchial tree, includes both wave speed and viscous mechanisms determining flow limitation and reproduces the respiratory mechanics of subjects undergoing mechanical ventilation.

The aim of the present paper is to modify this model by introducing lung nonhomogeneity in order to study thorough simulation what happens in a mechanically ventilated patient when only some regions of the lungs are affected by an obstructive pulmonary condition. In particular we intend to asses how the expiratory flow limitation phenomenon is influenced by pathological changes in affected airway portions and the effect of non-homogeneous airway obstruction on breathing mechanics. This effect will be evaluated by means of expiratory flow-volume curves, typically used to detect and quantify expiratory flow limitation in clinical practice.

\section{Methods}

\subsection{The new model}

The new model is based on the published homogeneous model [5] which is non linear, dynamic, and incorporates wave speed and viscous mechanisms determining expiratory flow limitation (EFL). The model was modified to study the effects of mechanical non-homogeneities in the EFL phenomenon. The new model is still based on Weibel's dichotomous morphometric description of the tracheobronchial tree [6]. The trachea (generation 0) forks into two main bronchi (generation 1) described separately and each bronchus gives rise to two distinct regions with the same number of branches. The resulting four regions $(\mathrm{AC}, \mathrm{AD}$, $\mathrm{BC}$ and $\mathrm{BD}$ ) have different geometrical and mechanical characteristics, and all branches of the same generation in a given region are assumed identical. Since generations from 3 to 16 are therefore represented symmetrically, it is possible to 
simplify their description as in the previous model, considering a given generation of each region to have $2^{\mathrm{n}-2}$ airways. The schematic representation of the tracheobronchial tree is shown in fig. 1 , where the two parameters $R_{p}$ and $C_{p}$ represent the overall resistance and compliance of the respiratory zone (generations 17-23) of each independent region.

With respect to the previous model, we modified the relationship between branch diameter and transmural pressure according to Lambert et al. [7]; in region $r$ the branch diameter of generation $n$ expressed as a percentage of its maximum value is:

$$
\begin{aligned}
& d_{b_{n, r}} \%=\left\{\begin{array}{l}
100 *\left(\alpha_{0_{n, r}} *\left(1-\frac{\alpha_{0_{n, r}}^{\prime} * P_{n, r}}{\alpha_{0_{n, r}} * n_{1_{n, r}}}\right)^{-n_{1_{n, r}}}\right)^{0.5}, P_{n, r}<0 \\
100 *\left(1-\left(1-\alpha_{0_{n, r}}\right) *\left(1-\frac{\alpha_{0_{n, r}}^{\prime} * P_{n, r}}{\left(\alpha_{0_{n, r}}-1\right)^{*} n_{2_{n, r}}}\right)^{-n_{2, r}}\right)^{0.5}, P_{n, r} \geq 0 \\
\alpha_{0_{n, r}}=\left(\text { Area }_{b_{n, r}} / \text { Area }_{b_{n, r \max }}\right)_{P_{n, r}=0} ; \alpha_{0_{n, r}}^{\prime}=\left(d \alpha_{n, r} / d P_{n, r}\right)_{P_{n, r}=0} .
\end{array}\right.
\end{aligned}
$$

where Area $_{b n, r}$ and Area $_{b n, r \text { max }}$ are the branch area of generation $n$ in region $r$ and its maximum value, respectively, and $\alpha_{n, r}=$ Area $_{b, r} /$ Area $_{b n, r \max } . P_{n, r}$ is the transmural pressure of generation $n$ in region $r$. The five parameters $\left(\alpha_{0 n, r}, \alpha^{\prime}{ }_{0 n, r}\right.$, $n_{1 n, r}, n_{2 n, r}$, Area $\left._{b, r \text { max }}\right)$ specify the diameter-transmural pressure curve of each generation.

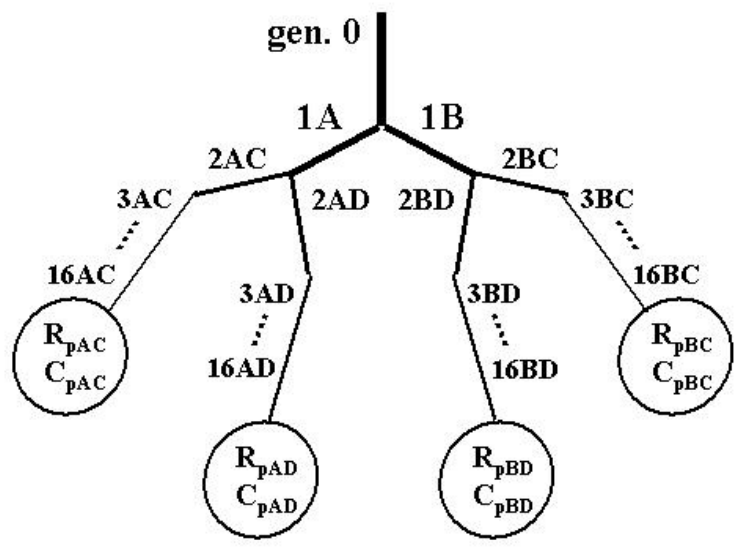

Figure 1: $\quad$ Schematic non-homogeneous structure of the tracheobronchial tree. 
Branch compliance can be easily obtained from the new relationship between branch diameter and transmural pressure [eqn. (1)] and from eqns. (3) and (5) of the homogeneous model [5]. Moreover equations characterizing resistance and inertance of each branch are analogous to eqns (1) and (2) of the homogeneous model [5], respectively, considering generation $n$ of region $r$ instead of the generic generation $n$ (subscript $n, r$ instead of $n$ ). Identically the total pressure drop over the $\mathrm{n}^{\text {th }}$ generation of region $r$ can be obtained from eqn. (6) of the homogeneous model [5].

\subsection{Simulation setting}

Normal respiratory conditions were simulated using transmural pressurediameter curves similar to the curves proposed by Lambert et al. [7] and assuming respiratory zone resistance and compliance of each region of $6 \mathrm{cmH}_{2} \mathrm{O} \mathrm{l}^{-1} \mathrm{~s}$ and $0.021 \mathrm{cmH}_{2} \mathrm{O}^{-1}$, respectively.

In order to reproduce a severely obstructive condition the respiratory zone resistance of each region was doubled and maximum airway diameter of generations 8-16 was halved. The transmural pressure-diameter curves of generations 8-16 (see fig. 2) were also modified to account for an easier collapsibility of these airways. Pathological changes were applied to all four regions in like manner.


Figure 2: Branch diameter, as a percentage of its maximum value, plotted against transmural pressure for airways of the conductive zone of a generic region. Top: normal case; bottom: severe pathological case. 
Parameters of the transmural pressure-diameter curve of a generic region are listed in table 1 for normal (normal) and severe pathological (pathol.) cases. The values of other parameters of the new model were not modified with respect to the homogeneous model [5], nor was ventilator setting changed.

In order to analyze what happens when only a part of lungs is obstructed, we considered two different situations:

Situation $a$ ) the above pathological changes only affect a percentage of the lungs (region $\mathrm{AC}$ in case $a 1$, i.e. $25 \%$; regions $\mathrm{AC}$ and $\mathrm{AD}$ in case $a 2$, i.e. $50 \%$; regions $\mathrm{AC}, \mathrm{AD}$ and $\mathrm{BC}$ in case $a 3$, i.e. $75 \%$ ), while the other parts of the lungs are normal;

Situation $b$ ) the pathological changes in transmural pressure-diameter curves affect all four regions, while pathological changes in respiratory zone resistance and in maximum airway diameter only affect a percentage of the lungs (region $\mathrm{AC}$ in case $b 1$, i.e. $25 \%$; regions $\mathrm{AC}$ and $\mathrm{AD}$ in case $b 2$, i.e. $50 \%$; regions $\mathrm{AC}, \mathrm{AD}$ and $\mathrm{BC}$ in case $b 3$, i.e. $75 \%$ ).

Table 1: Model parameters of the transmural pressure-diameter curve of the conducting zone in a generic region $r$ of a normal (normal) and a severe pathological (pathol.) case.

\begin{tabular}{|l|l|l|l|l|l|l|l|l|}
\hline $\begin{array}{l}\text { Gen. } \\
\text { n. }\end{array}$ & $\begin{array}{l}\alpha_{0} \\
\text { normal }\end{array}$ & $\begin{array}{l}\alpha_{0}^{\prime} \\
\text { normal } \\
\mathrm{cmH}_{2} \mathrm{O}^{-1}\end{array}$ & $\begin{array}{l}n_{1} \\
\text { normal }\end{array}$ & $\begin{array}{l}n_{2} \\
\text { normal }\end{array}$ & $\begin{array}{l}\alpha_{0} \\
\text { pathol. }\end{array}$ & $\begin{array}{l}\alpha_{0}^{\prime} \\
\text { pathol. } \\
\mathrm{cmH}_{2} \mathrm{O}^{-1}\end{array}$ & $\begin{array}{l}n_{l} \\
\text { pathol. }\end{array}$ & $\begin{array}{l}n_{2} \\
\text { pathol. }\end{array}$ \\
\hline 1 & 0.882 & 0.0294 & 1 & 10 & 0.882 & 0.0294 & 1 & 10 \\
\hline 2 & 0.686 & 0.05 & 1 & 10 & 0.686 & 0.05 & 1 & 10 \\
\hline 3 & 0.550 & 0.0784 & 1 & 10 & 0.550 & 0.0784 & 1 & 10 \\
\hline 4 & 0.495 & 0.098 & 1 & 10 & 0.495 & 0.098 & 1 & 10 \\
\hline 5 & 0.445 & 0.1225 & 1 & 10 & 0.445 & 0.1225 & 1 & 10 \\
\hline 6 & 0.410 & 0.1392 & 1 & 10 & 0.410 & 0.1392 & 1 & 10 \\
\hline 7 & 0.385 & 0.1559 & 1 & 10 & 0.385 & 0.1559 & 1 & 10 \\
\hline 8 & 0.362 & 0.1706 & 1 & 10 & 0.164 & 0.2686 & 83.04 & 1.12 \\
\hline 9 & 0.346 & 0.1804 & 1 & 10 & 0.125 & 0.2686 & 83.04 & 1.12 \\
\hline 10 & 0.334 & 0.1902 & 1 & 10 & 0.095 & 0.2686 & 83.04 & 1.12 \\
\hline 11 & 0.324 & 0.2020 & 1 & 9 & 0.071 & 0.2686 & 83.04 & 1.12 \\
\hline 12 & 0.317 & 0.2137 & 1 & 8 & 0.054 & 0.0081 & 83.04 & 1.12 \\
\hline 13 & 0.310 & 0.2216 & 1 & 8 & 0.039 & 0.0081 & 83.04 & 1.12 \\
\hline 14 & 0.306 & 0.2284 & 1 & 8 & 0.030 & 0.0081 & 83.04 & 1.12 \\
\hline 15 & 0.302 & 0.2343 & 1 & 7 & 0.020 & 0.0081 & 83.04 & 1.12 \\
\hline 16 & 0.299 & 0.2382 & 1 & 7 & 0.010 & 0.0081 & 83.04 & 1.12 \\
\hline
\end{tabular}



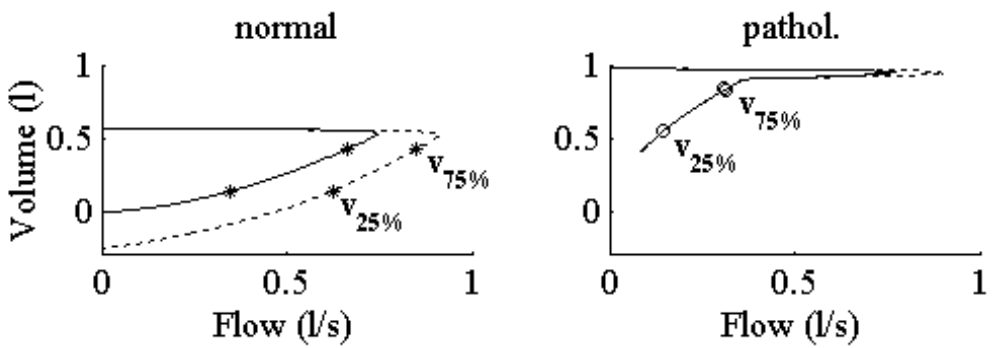

Figure 3: Flow-volume curve of test cycle (broken line) and preceding control cycle (continuous line) obtained by NEP technique. $\mathrm{v}_{75 \%}$ and $\mathrm{v}_{25 \%}$ are the last $75 \%$ and $25 \%$ of exhaled volume, respectively.
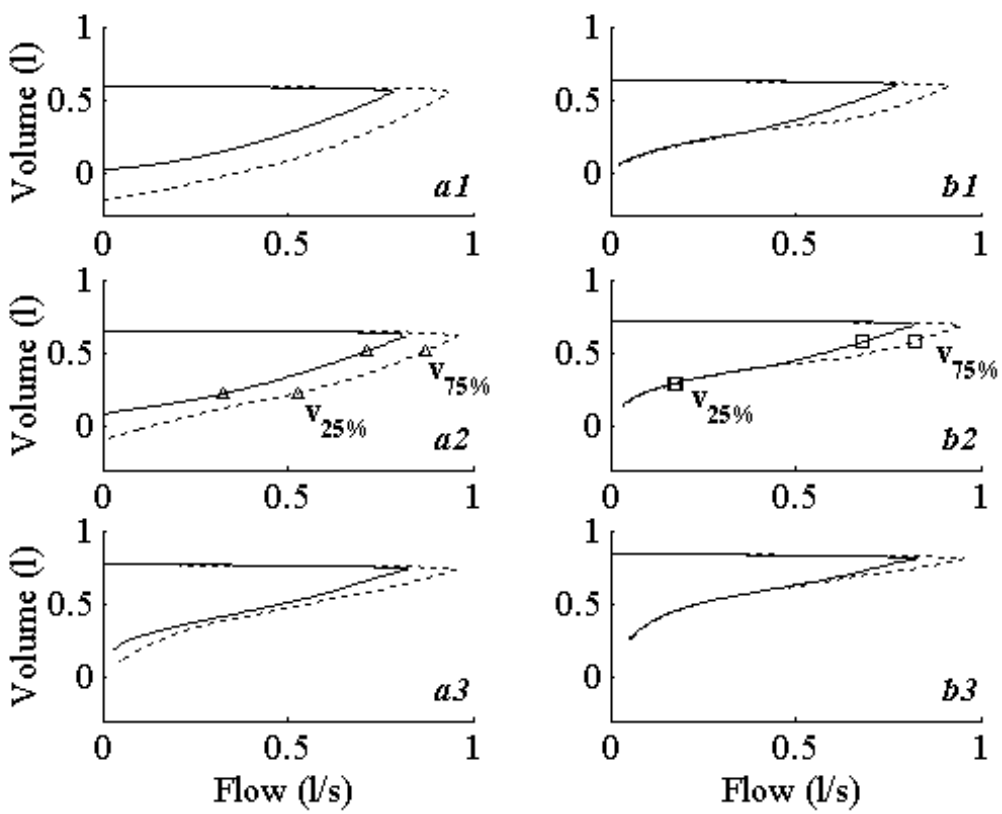

Figure 4: Flow-volume curve of test cycle (broken line) and preceding control cycle (continuous line) obtained by NEP technique in Situations $a$ and $b . \mathrm{v}_{75 \%}$ and $\mathrm{v}_{25 \%}$ are the last $75 \%$ and $25 \%$ of exhaled volume, respectively.

\section{Results}

The negative expiratory pressure (NEP) method [8] was used to detect EFL in all simulated cases. In particular a pressure of $-5 \mathrm{cmH}_{2} \mathrm{O}$ was applied to the 
expiratory circuit outlet at the beginning of expiration and was maintained until the end of expiration. We also computed the flow limited portion (FLP-NEP), expressed as a percentage of expiratory tidal volume in all cases [9].

Figure 3 shows the expiratory flow-volume curves in normal and severe pathological cases during a test cycle with NEP applied (broken line) and during the preceding control cycle (continuous line). It can be seen that EFL is present in the severe pathological case over most of expiration (FLP-NEP equal to about $88 \%$ ), while it is absent in the normal case.

Expiratory flow-volume curves were also analyzed in Situations $a$ and $b$ (see fig. 4). No EFL was observed in Situation $a$, whereas in Situation $b$ the test expiratory flow-volume curve was partially superimposed on the control curve (FLP-NEP about $42 \%, 48 \%$, and $66 \%$ in cases $b 1, b 2$, and $b 3$, respectively). To better analyze what happens when obstructive pulmonary condition varies, we chose four exemplary cases (normal, a2, b2 and pathol.) and examined the equivalent total radius of the generations of regions $\mathrm{AC}$ and $\mathrm{BD}$ in the conducting zone, i.e. the radius computed from the total cross sectional area of each generation of the considered region.

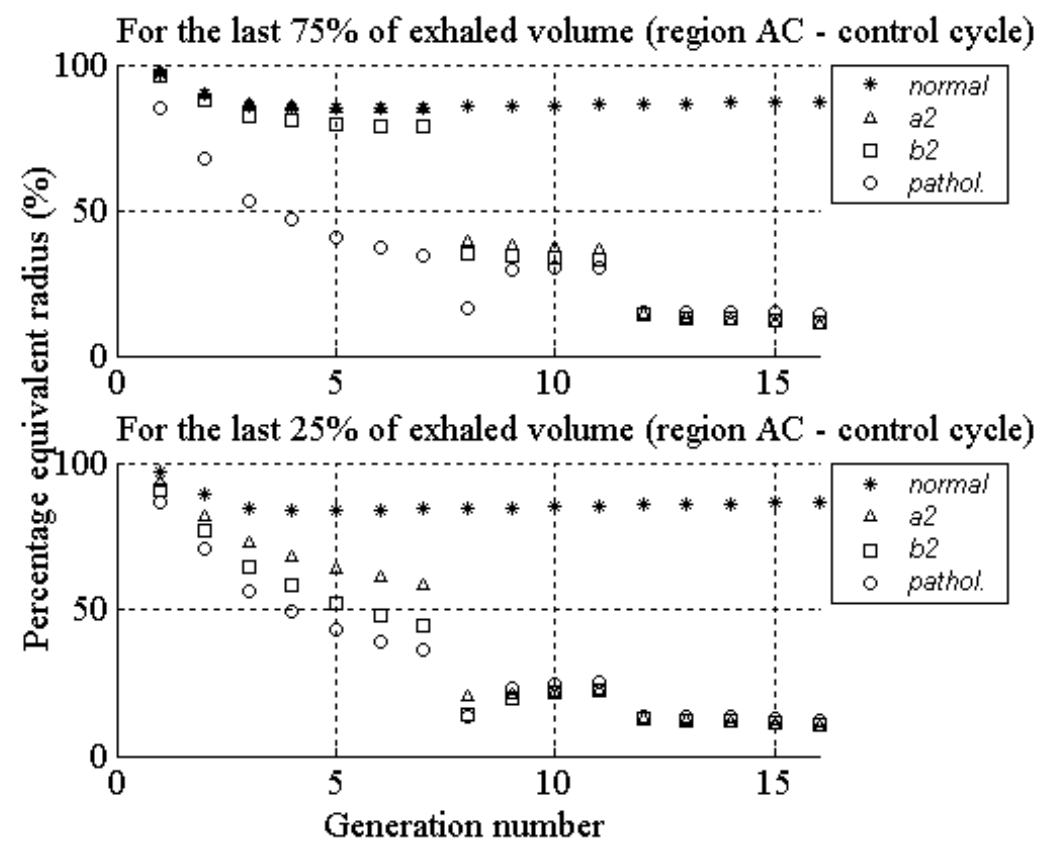

Figure 5: Equivalent radius vs. airway location in region $\mathrm{AC}$ for the examined cases during control cycle for the last $75 \%$ (upper part) and $25 \%$ (bottom part) of exhaled volume.

Figures 5 and 6 show the equivalent radii, expressed as a percentage of Weibel radii, as a function of generation number in regions $\mathrm{AC}$ and $\mathrm{BD}$, 
respectively. Equivalent radii were evaluated at the last $75 \%$ and $25 \%$ of exhaled tidal volume ( $\mathrm{v}_{75 \%}$ and $\mathrm{v}_{25 \%}$, respectively). Figure 5 shows a great reduction in equivalent radii of generations 8 to 16 in all pathological cases $(a 2, b 2$, pathol.) for $\mathrm{v}_{75 \%}$ and $\mathrm{v}_{25 \%}$, indicating collapse of these generations in region AC. Of course, in region BD (fig. 6), equivalent radii of generations 8 to 16 behaved as in $\mathrm{AC}$ in normal and pathol. cases, because of system homogeneity. On the contrary, in case $a 2$ they behaved as in the normal case for $\mathrm{v}_{75 \%}$ and they slightly decreased for $\mathrm{v}_{25 \%}$. In case $b 2$ the equivalent radii of the same generations were similar to those of the severe pathological case for $\mathrm{v}_{25 \%}$, whereas for $\mathrm{v}_{75 \%}$ only the equivalent radius of lower generations (from 12 to 16) showed a significant reduction. In all four regions the equivalent radius of generations 1 to 7 decreased for $\mathrm{v}_{75 \%}$ and $\mathrm{v}_{25 \%}$ in the severe pathological case, whereas in cases $a 2$ and $b 2$ the reduction was only evident for $\mathrm{v}_{25 \%}$.



Figure 6: Equivalent radius vs. airway location in region $\mathrm{BD}$ for the examined cases during control cycle for the last $75 \%$ (upper part) and $25 \%$ (bottom part) of exhaled volume.

\section{Discussion and conclusions}

Although the pathological condition reproduced in situation $a 2$ causes a collapse of generations 8 to 16 in half the lungs (fig. 5), the expiratory flow-volume curve 
in the control cycle is quite similar to the normal curve and the NEP method does not highlight EFL in any part of expiration (fig. 4). A similar situation is also found in cases $a 1$ and $a 3$. With respect to the normal case, however, Situation $a$ shows an increase in maximum tracheal pressure, e.g. maximum pressure of case $a 2$ is about $49 \%$ greater than the normal value (data not shown). In Situation $b$ lower generations show a great reduction in equivalent radius in both lungs (see regions $\mathrm{AC}$ and $\mathrm{BD}$ in figs. 5 and 6, respectively), but EFL does not develop until collapse also involves the lower part of the intermediate airways (i.e. generations 8 to 11 ) in both lungs (fig. $4, v_{25}$ ). In fact when the equivalent radius of these generations is reduced in part of the lungs, the control and test curves are not superimposed (fig. $4, \mathrm{v}_{75} \%$ ).

In conclusion, in the situations examined, EFL only seems to develop if the collapse involves all lung regions, albeit in different manners, and if the intermediate airways become significantly collapsible. Moreover, it seems impossible to determine the presence of non-homogeneities in the lung or to detect collapse of a single part of the lung from analysis of expiratory flowvolume curves.

\section{Acknowledgment}

This work was supported by the Italian Ministry of Education, University and Research (MIUR).

\section{References}

[1] Lambert, R.K., Simulation of the effects of mechanical nonhomogeneities on expiratory flow from human lungs. J. Appl. Physiol., 68(6), pp. 25502563, 1990.

[2] Gillis, H.L. \& Lutchen, K.R., How heterogeneous bronchoconstriction affects ventilation distribution in human lungs: a morphometric model. Ann. Biomed. Eng., 27(1), pp. 14-22, 1999.

[3] Nucci, G., Suki, B. \& Lutchen, K.R., Modeling airflow-related shear stress during heterogeneous constriction and mechanical ventilation. J. Appl. Physiol., 95(1), pp. 348-356, 2003.

[4] Polak, A.G. \& Lutchen, K.R., Computational model for forced expiration from asymmetric normal lungs. Ann. Biomed. Eng., 31(8), pp. 891-907, 2003.

[5] Barbini, P., Brighenti, C., Cevenini, G. \& Gnudi, G., A dynamic morphometric model of the normal lung for studying expiratory flow limitation in mechanical ventilation. Ann. Biomed. Eng., 33(4), pp. 518-530, 2005.

[6] Weibel, E.R., Morphometry of the human lung, Springer-Verlag: Berlin, 1963.

[7] Lambert, R.K., A new computational model for expiratory flow from nonhomogeneous human lungs. J. Biomech. Eng., 111(3), pp. 200-205, 1989. 
[8] Valta, P., Corbeil, C., Lavoie, A., Campodonico, R., Koulouris, N., Chasse, M., Braidy, J. \& Milic-Emili, J., Detection of expiratory flow limitation during mechanical ventilation. Am. J. Respir. Crit. Care Med., 150(5Pt1), pp. 1311-1317, 1994.

[9] Lourens, M.S, Berg, B.V., Hoogsteden, H.C. \& Bogaard, J.M., Detection of flow limitation in mechanically ventilated patients. Intensive Care Med., 27(8), pp. 1312-20, 2001. 